\title{
Study of Sterilization Effects on Marine Vibrio sp. using Interaction of Cavitation with Shock Wave in a Narrow Water Chamber
}

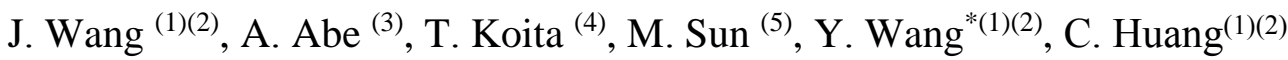

\begin{abstract}
When underwater shock waves are generated by an electric discharge in a narrow water chamber, the instant release of a great amount of energy causes the propagation of a shear wave in wall material with the deformation of the chamber wall. The shear waves produce decompression in water and result in the growth of bubble nuclei. Subsequently, those oscillating cavitation bubbles are exposed to the shock pressures, and thus free radicals and rebound shock waves are generated due to their violent collapses. Eventually, marine bacteria around them are inactivated by these productions. In the present study, we investigate the sterilization effects of these oscillating bubbles and cavitation-shock interaction by bio-experiments, respectively. Furthermore, the chemical action of free radicals on marine bacteria is discussed. The generation of the $\mathrm{OH}$ radicals is clarified by measuring the concentration of the $\mathrm{H}_{2} \mathrm{O}_{2}$. To estimate the generation condition of the $\mathrm{OH}$ radicals, a bubble dynamic model consisting of an oscillation model for the growth of bubble nuclei and an impact model to describe the cavitation-shock interaction is developed. Finally, the theoretical estimation by the bubble dynamic model is discussed under the conditions of the present experiments.
\end{abstract}

Keywords: Cavitation-shock interaction, Sterilization effects, Bubble dynamic model, OH radicals, Narrow water chambers

\section{Introduction}

The phenomenon of cavitation was first discovered in 1894 when the tests were made to determine why a ship could not reach its design speed during sea trials. It was found that the generated cavitation makes the hydrodynamic performance of a propeller reduce, while also give rise to vibration and erosion. After that, cavitation bubbles have been observed in many different fields, and their dynamic behaviors have been studied in detail experimentally, theoretically, and numerically. In experiment, there are some generation methods of cavitation bubbles, such as hydrodynamic method including hydraulic and ultrasonic cavitation, and liquid breakdown induced by laser, electric discharge, or underwater explosion. When local static pressure in liquid decreases rapidly below a limiting pressure, the hydrodynamic cavitation occurs and finally would develop into cloud cavitating flow, such as the flows of hydraulic machinery of pump, screws, and water turbines. The collapse and shedding of the cavitation would bring destructive damage to the machinery (1-4). However, the destruction also has a positive effect with appropriate controlling techniques, in the fields of medical therapy, drug delivery, food engineering, waste water treatment, and so on. Takayama (5) found the liquid jet induced by the motion of cavitation bubble near body stone could enhance the effect of extracorporeal shock wave lithotripsy. These cavitation bubbles were generated in tensile region behind underwater shock wave focusing. For drug delivery, the controlled motion of cavitation bubbles by acoustics were proven to enhance drug activity and uptake when high intensity focused ultrasound was introduced to develop a novel technique of targeting drugs to tumors (6). Song et al. (7) applied a high-power laser to the cleaning of the solid surface in liquid and pointed out that a high cleaning efficiency for the removal of particles was obtained by using liquid jets and rebound shock waves induced during 
the bubble collapse. Loske et al. (8) developed a non-thermal food preservation method using the cavitation-shock interaction and clarified the bactericidal effect of Escherichia coli in an electrohydraulic shock wave generator. And they indicated that the bacteria were inactivated by the mechanical action of the shock wave. In the field of maritime sciences, the collapse of microbubble was used to the sterilization of ships' ballast water (9). When they carried out a bioexperiment to investigate the sterilization effect of the shock wave-microbubble interaction, Wang and Abe (10) found the cavitation bubbles generated behind the concentration of underwater shock waves have a potential to inactivate marine bacteria.

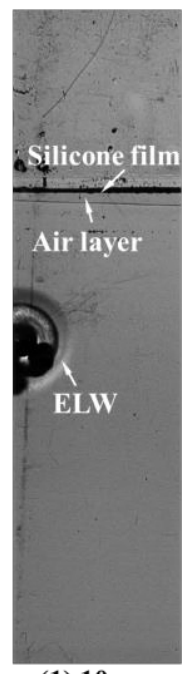

(1) $10 \mu \mathrm{s}$

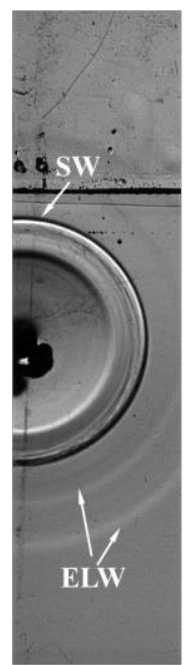

(2) $20 \mu \mathrm{s}$

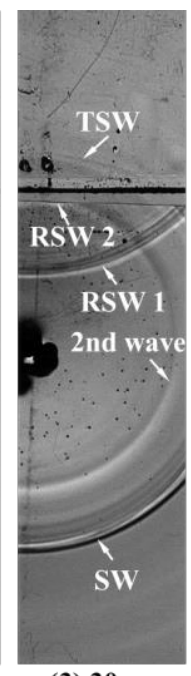

(3) $30 \mu \mathrm{s}$

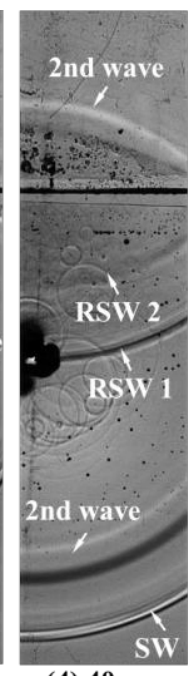

(4) $40 \mu \mathrm{s}$

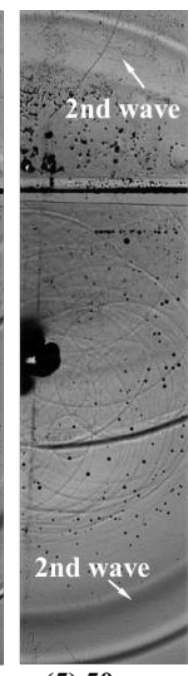

(5) $50 \mu \mathrm{s}$

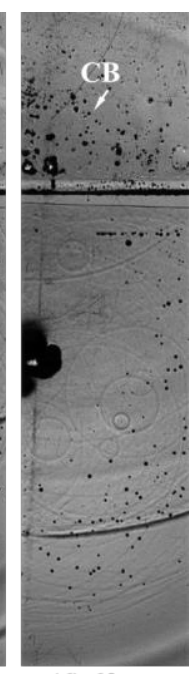

(6) $60 \mu \mathrm{s}$

Fig. 1 Observation of multiple waves generated by underwater electric discharge with 2-mm air layer in Ref. (12). ELW: Elastic wave, SW: Shock wave, RSW1: Reflection of shock wave,

RSW: Reflection of 2nd wave, TSW: Transition of shock wave through air layer, and CB:

\section{Cavitation bubbles.}

On the other research, Koita et al. (11) observed the generation of cavitation bubbles behind the propagation of multiple waves produced by underwater electric discharge in a narrow water chamber. Regarding the reason of the cavitation generation, they argued that the interactions of the waves between the outer and inner interface of the acrylic wall of the chamber caused decompression in water. To clarify the generation mechanism of these cavitation bubbles, Wang et al. (12) carried out an experiment by producing an air layer between a bag made by silicone film and the water surface in the narrow water chamber in order to remove the action of underwater shock waves, as shown in Fig. 1. The figure shows that a weak transmitted shock wave (TSW) is captured at $30 \mu$ s although the propagation of an underwater shock wave is intercepted well by the air layer. Subsequently, a 2nd wave is propagating in the upper side of the air layer and cavitation bubbles are generated behind the 2 nd wave. They argued that the 2 nd wave was not induced by the permeation of underwater shock wave. On the other hand, as shown in Fig. 1, the elastic wave (ELW) propagating in the wall material is in front of the shock wave (SW) so that the 2nd wave is also not from the reflection of the ELW. They concluded that the 2nd wave causing the generation of cavitation bubbles was a shear wave travelling in the window material. The wave was induced by the deformation of the wall material when large amounts of energy were released as a result of underwater electric discharge. In addition, they also found that interaction of cavitation bubbles with shock waves could enhance the inactivation effect on marine bacteria. Cavitation bubble could be also called as inertial bubble because of its oscillation in the absence of external pressure loading (13-15). Klaseboer et al. (16) investigated the dynamic interaction of a pressure pulse with laser-induced inertial bubble experimentally and numerically, 
respectively. Their results clarified that the more intense collapses of the bubbles occurred due to the action of shock pressure.

From those backgrounds, considering the sterilizing potential of cavitation bubbles, it is interesting to understand the inactivation effect of the cavitation-shock interaction on bacteria in a narrow water chamber. In bio-experiments of marine Vibrio sp., underwater shock waves are produced by a high-voltage power supply, and the cell suspension is isolated from distilled water in the chamber by using a silicone bag. In the experiments, optical observation and pressure measurement are simultaneously conducted to analyze the propagation of underwater shock waves and behaviors of cavitation bubbles. To investigate the sterilization effects of only these oscillating bubbles, an air layer is set to prevent underwater shock waves directly passing through the cell suspension. The respective effects of the chemical and mechanical action of the bubble collapses are also examined. Furthermore, the generation of the $\mathrm{OH}$ radicals is clarified by measuring the concentration of the $\mathrm{H}_{2} \mathrm{O}_{2}$. On the other hand, a bubble dynamic model consisting of an oscillation model and an impact model is developed to investigate the condition for generating the $\mathrm{OH}$ radicals. Finally, we discuss theoretical solution of the bubble dynamic model under the condition of the present experiments.

\section{Bio-experimental setup}

Figure 2 shows a schematic of the bio-experimental setup in a narrow water chamber. The dimensions of the narrow water chamber were $300 \mathrm{~mm}(\mathrm{H}) \times 240 \mathrm{~mm}(\mathrm{~W}) \times 5 \mathrm{~mm}(\mathrm{D})$. Given the observation by Koita et al. (11), it was found that the area at which cavitation bubbles were generated was an annular at a position of about $20 \mathrm{~mm}$ from the discharge point. To examine the sterilization effects of these cavitation bubbles, a bag made of a $0.1-\mathrm{mm}$ silicone film was designed in the water chamber and filled with cell suspension of marine Vibrio sp., as shown in Fig. 2. The dimensions of the silicone bag were $120 \mathrm{~mm}(\mathrm{H}) \times 100 \mathrm{~mm}(\mathrm{~W}) \times 5 \mathrm{~mm}$ (D). Its acoustic impedance is almost the same as that of water. The discharge point was set up at a distance of about $23 \mathrm{~mm}$ from the bottom of the silicone bag in the water chamber. Underwater shock waves were continuously generated from the discharge point by a high-voltage pulse power supply (HPS 18K-A, Tamaoki Electronics Co-Ltd) and a pulse generator. In the experiments, the water chamber was filled with distilled water to make sure the triggers of the electric discharges. Its applied frequency was $1 \mathrm{~Hz}$. In addition, optical visualization of schlieren method and pressure measurements were conducted to analyze the propagation behaviors of shock wave and collapsing motion of cavitation bubbles in the water chamber.

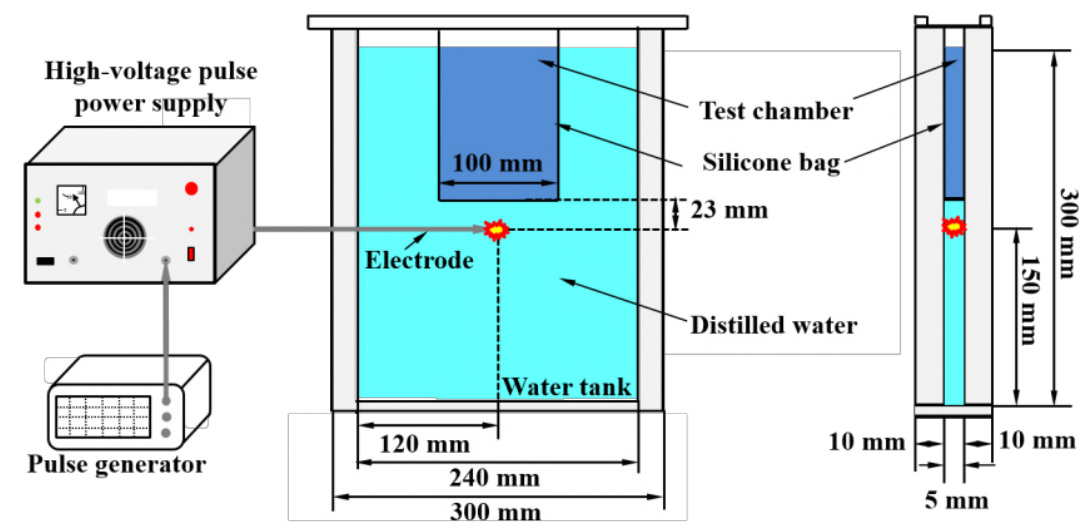

Fig. 2 Schematic of bio-experimental setup for estimating sterilization effects 
Figure 3 illustrates a considerable sterilization mechanism in the narrow water chamber. Cavitation bubbles are generated behind a shear wave propagating the wall material due to instant release of large amount energy at the discharge point. After the underwater shock waves from the reflection on the chamber wall or the transmission of the elastic wave pass through these oscillating bubble, the intense collapses are induced with the generation of rebound shock waves and free radicals that inactivate marine bacteria by the mechanical and chemical action, respectively. A photo of marine Vibrio sp. used in the bio-experiments is shown in Fig. 3 (c). For estimating the sterilization effects of the cavitation-shock wave interaction, cell experiments were carried out in the silicone bag. Samples were extracted regularly from the cell suspension, diluted serially, and spread on the agar plate. The agar places were incubated for 24 hours at $35^{\circ} \mathrm{C}$. The cell viability in $1 \mathrm{ml}$ was evaluated using the number of colony-forming cell in the agar places on the basis of the dilution ratio.

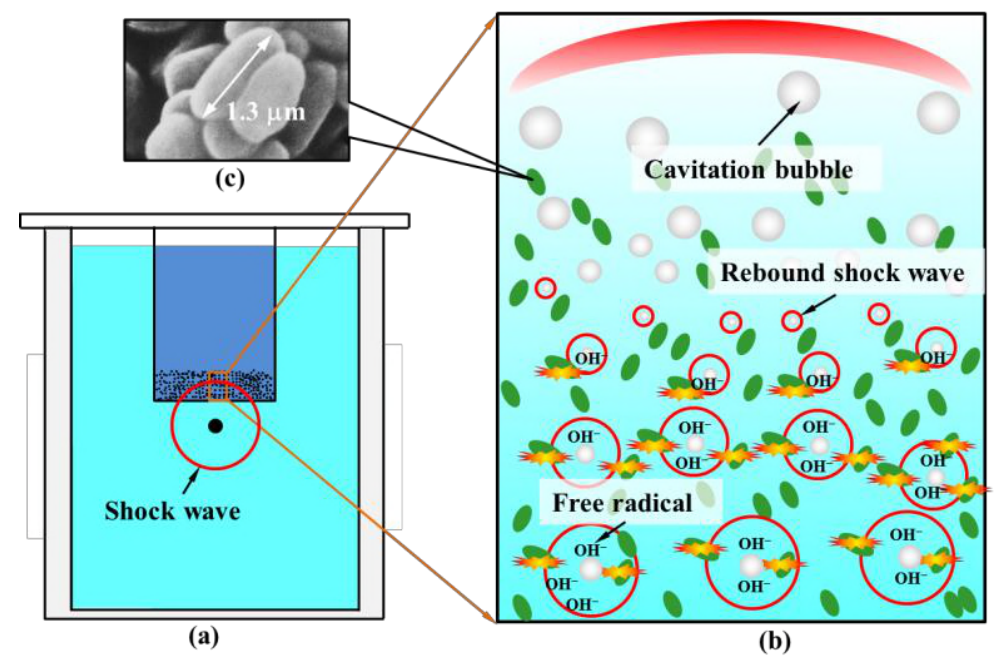

Fig. 3 Schematic of inactivation mechanism of cavitation-shock interaction generated in narro w water chamber: (a) Schematic of narrow water chamber, (b) Collapses of cavitation bubbles, a nd (c) Photo of marine Vibrio sp.

\section{Bubble dynamic model}

According to our previous study (17), the action of the $\mathrm{OH}$ radicals is mainly responsible for inactivating marine bacteria in a cylindrical water chamber at a $31.6-\mathrm{kV}$ electric discharge. Comparing with the experimental conditions in Ref. (17), the shock pressures inducing bubble collapse in the narrow water chamber are obviously lower, especially in the case of setting an air layer, as shown in Fig. 1. Nevertheless, we could obtain a high sterilization effect in Ref. [12], which is thought to depend on the biochemical action of the $\mathrm{OH}$ radicals. Consequently, a bubble dynamic model was developed for investigating the condition for the generation of the $\mathrm{OH}$ radicals in the interaction of cavitation bubbles and shock pressures, as shown in Fig. 4. From the given generation mechanism, these cavitation bubbles are generated from bubble nuclei when local static pressure is decompressed below the saturated vapor pressure by the tensile action in water with the propagation of a shear wave in the wall material. After that, these bubbles are exposed to the pressure oscillations by reflected shock waves in water. To analyze those collapsing motion, it requires to build an oscillation model for the growth of bubble nuclei and an impact model for the cavitation-shock interaction in the bubble dynamic model. In the oscillation model, the growth of bubble nuclei is thought to be isothermal since the heat transfer is fast 
relative to the time scale of the bubble motion (18). In the meantime, an equilibrium evaporation and condensation are also considered to investigate the transportation of water vapor at the bubble interface. For the cavitation-shock interaction, it is necessary to analyze the bubble motion after the bubble in arbitrary motion phase is exposed to a shock pressure, as shown in the figure. Here, the heat transfer through a thermal boundary layer is needed to estimate the conditions for generating the $\mathrm{OH}$ radicals besides the transportation of water vapor. In the bubble dynamic model, the bubble is assumed to maintain a spherical shape during its collapsing motion.

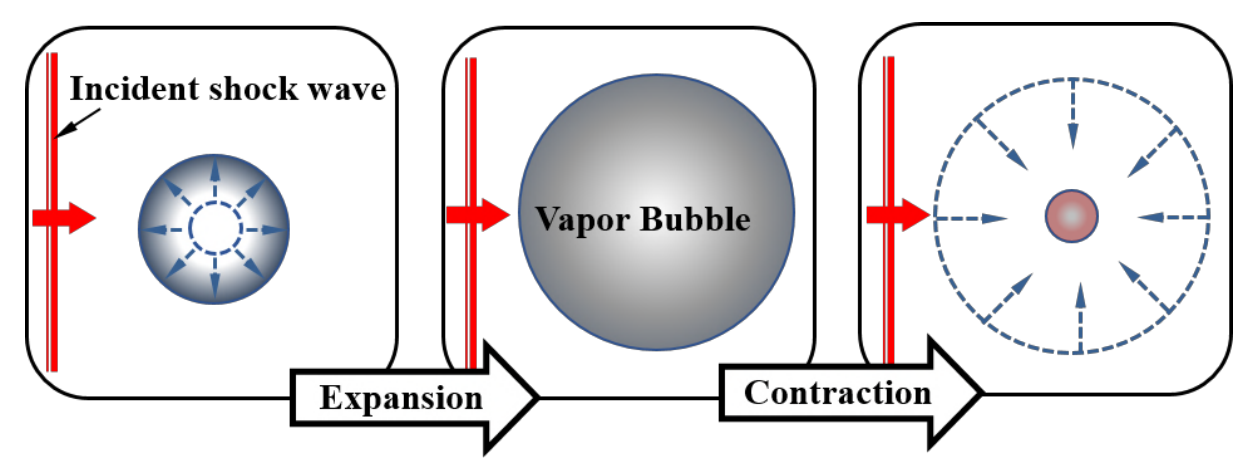

Fig. 4 Schematic of bubble dynamic model for describing growth of bubble nuclei and cavitation-shock interaction

\subsection{Oscillation model}

In the model, the Rayleigh-Plesset equation was solved to describe the growth of bubble nuclei, as presented in Eq. (1).

$$
R \ddot{R}+\frac{3}{2} \dot{R}^{2}=\frac{1}{\rho}\left(P_{\text {in }}-P_{\infty}-\frac{4 \mu \dot{R}}{R}-\frac{2 \sigma}{R}\right) .
$$

where $R$ is the bubble radius, $\dot{R}=\mathrm{d} R / \mathrm{d} t, t$ is the time, $\ddot{R}=\mathrm{d} \dot{R} / \mathrm{dt}, P$ in is the pressure inside the bubble, $P_{\infty}$ is the pressure behind an incident shock wave, $\sigma$ is the surface tension, and $\mu$ is the viscosity coefficient.

For the equation for state inside the bubble, the van der Waals equation was used,

$$
P_{\text {in }} v=R_{g} T_{\text {in }}
$$

where $v$ and $T_{\text {in }}$ are the molar volume and temperature of the gas inside the bubble, and $R_{\mathrm{g}}=$ $8.3145 \mathrm{~J} /(\mathrm{mol} \mathrm{K})$. The molar volume $v$ is presented by,

$$
v=N_{A} V / N_{\text {Tot }}
$$

where $N_{\mathrm{A}}$ is the Avogadro number, $6.02 \times 10^{23}$, the volume of the bubble $V=4 / 3 \pi R^{3}$, and $N_{\text {Tot }}$ is the total number of gas molecules in the bubble, consisting of the number of water vapor $N_{\text {water }}$ and other molecules $N_{\text {others. }}$

$$
N_{\text {Tot }}=N_{\text {water }}+N_{\text {others }} \text {. }
$$

In the model, we just consider the transfer of water vapor through a boundary layer during the growth of a nucleus. Given the study of Toegel et al. (19), the rate of particle change for water vapor can be estimated by Eq. (5).

$$
\dot{N}_{\text {water }} \approx 4 \pi R^{2} D \frac{C_{r}-C}{l_{\text {diff }}}
$$

where $C_{\mathrm{r}}=C_{\mathrm{r}}\left(T_{0}\right)$ corresponds to the equilibrium density of water vapor at the bubble wall (20, 21), $C_{\mathrm{r}}=P_{\mathrm{v}}\left(T_{0}\right) / k T_{0} \approx 5.9 \times 10^{23} \mathrm{~m}^{-3}$ for $T_{0}=293.15 \mathrm{~K}, k$ is the constant, $1.38 \times 10^{-23}(22), C$ is the actual concentration of water vapor inside the bubble, $C=N_{\text {water }} / V, D$ is the diffusion constant, and $l_{\text {diff }}$ is the diffusive penetration depth. In the study of Toegel et al. (22), the diffusive 
penetration depth can be presented referring to the expression of a thermal boundary layer. Hence, $l_{\text {diff }}$ could be obtained with the Rayleigh-Plesset time $\tau_{\mathrm{c}}(17,23)$.

$$
l_{\text {diff }}=\sqrt{D \tau_{c}}=\sqrt{D 0.915 R_{0} \sqrt{\frac{\rho_{l}}{p_{\infty}-P_{0}}}}=\sqrt{\left(\lambda_{g} / \rho_{g} C_{p}\right) 0.915 R_{0} \sqrt{\frac{\rho_{l}}{p_{\infty}-P_{0}}}}
$$

where the diffusive constant $D$ is approximately equals to the thermal diffusivity $\alpha_{\mathrm{g}}=\lambda_{\mathrm{g}} / C_{\mathrm{p}} \rho_{\mathrm{g}}, \lambda_{g}$ is the thermal conductivity, $C_{\mathrm{p}}$ the heat capacity at constant pressure, $P_{0}$ is the atmospheric pressure, $\rho_{\mathrm{g}}$ is the density of gas and varies with the temperature and pressure inside the bubble, as shown in Eq. (7).

$$
\rho_{g}=\frac{m_{\text {Tot }}}{v \times 10^{3}}=\frac{\left(m_{\text {others }} \times N_{\text {others }}+m_{\text {water }} \times N_{\text {water }}\right)}{v \times 10^{3}}
$$

where $m$ denotes the molar mass in unit of $\mathrm{g} / \mathrm{mol}$.

\subsection{Impact model}

In the impact model, the Herring bubble equation was applied considering the compressible of water when the speed of sound was assumed to be constant (24).

$$
\left(1-\frac{2 \dot{R}}{C_{\infty}}\right) R \ddot{R}+\frac{3}{2}\left(1-\frac{4}{3} \frac{\dot{R}}{C_{\infty}}\right) \dot{R}^{2}+\frac{1}{\rho_{\infty}}\left(P_{\infty}-P_{s}-\frac{R}{C_{\infty}} \frac{d P_{s}}{d t}\right)=0,
$$

where $C_{\infty}$ is the sound speed of water at infinity, $\rho_{\infty}$ is the density of water at infinity, and $P_{\mathrm{s}}$ is the pressure at the wall of a bubble.

$C_{\infty}$ is given by

$$
C_{\infty}=\sqrt{\frac{n\left(P_{\infty}+B\right)}{\rho_{\infty}}},
$$

where $B$ and $n$ are constant values, $B=2963$ bar and $n=7.41$.

$P_{s}$ is described by

$$
P_{s}=P_{\text {in }}-\frac{1}{R}(2 \sigma+4 \mu \dot{R}) \text {, }
$$

where $P_{\text {in }}$ is the pressure of the gas inside the bubble.

Besides the transportation of water vapor through the diffusive penetration depth, as expressed in Eqs (4)-(7), the effect of the thermal conduction at the bubble wall was also required in the impact model. Hence, the energy balance of the gas inside the bubble is written in Eq. (11) according to the first law of the thermodynamics, where the bubble radius varies from $R$ to $R+\Delta R$ and the gas temperature changes by $\Delta T$ during the time $\Delta t$ :

$$
\left(\frac{4}{3} \pi R^{3}\right) \rho_{g} C_{v g} \Delta T=-\left(\Delta Q+P_{S}\left(4 \pi R^{2} \Delta R\right)\right)
$$

where $C_{\mathrm{vg}}$ is the specific heat of gas at constant volume. The term on the left-hand side presents the variation of the internal energy of the gas during the time $\Delta t$, the second term on the righthand side is the work of the pressure force at the bubble surface, and $\Delta Q$ is the heat released from the bubble to the liquid through a thermal boundary layer. The boundary layer is basically formed in the gas inside the bubble since the density and specific heat of water are so much larger than the respective values for gas. In the model, it is assumed that the temperature is spatially uniform at the center of the bubble, while to be linear within the boundary layer. Hence, $\Delta Q$ can be written in Eq. (12) according to the Fourier's law,

$$
\Delta Q \approx \frac{\lambda_{g}\left(4 \pi R^{2}\right) \Delta T}{\delta} \Delta t=\frac{\lambda_{g}\left(4 \pi R^{2}\right)\left(T_{i n}-T_{0}\right)}{\delta} \Delta t
$$


where $\delta$ is the thickness of the thermal boundary layer, $\delta \approx l_{\text {diff, as mentioned above. } T_{0} \text { and } T_{\text {in }}}$ are the temperature of the gas inside the bubble at $t=0$ and $t+\Delta t$.

Finally, the Rayleigh-Plesset and Herring bubble equation were solved using the fourth-order accurate Runge-kutta-Gill method

\section{Results and discussion}

Figure 5 shows multiple waves generated by an underwater electric discharge in the narrow water chamber, observed using the schlieren method. The output power of the electric discharge was $28.6 \mathrm{kV}$. The optical schlieren method was carried out using a metal halide lamp (LS-M350, SUMITA optical glass Inc.). The high-speed camera (i-SPEED 7, Nac Image Technology) captured images at a frame rate of $100 \mathrm{kfps}$ and an exposure time of $300 \mathrm{~ns}$. The resolution of the images was $840 \times 216$ pixels. In the figure, a 1st shock wave $(\mathrm{SW})$ generated by the electric discharge is observed in Fig. 5 (1). The 1st SW is thought to be cylindrical due to the thickness of the water chamber. The images indicate that its propagation speed is about $1500 \mathrm{~m} / \mathrm{s}$. At $20.87 \mu \mathrm{s}$, the 1 st SW is reflected partly at the bottom of the silicone bag, as indicated by Reflection 1, and then transmitted through the silicone film. After a wave passes through the film, an obscure outline of its reflected wave (Reflection 2) is also observed. According to our previous observation (12), the wave is thought to an elastic shear wave (SHW) generated by the deformation of the wall material due to the instant release of enormous energy when the electric discharge was triggered. From Fig. 5, it can be seen that cavitation bubbles (CB) are generated and grows behind the SHW in the upper side of the silicone film. Subsequently, rebound shock waves (CSW) are captured when the reflected shock waves (RSW) from the both sides of the water chamber pass through these cavitation bubbles, as shown in Fig. 5 (7). These observations indicate the inactivation of marine bacteria is expected in the cavitation-shock interaction. In addition, the pressure measurement of FOPH 2000 (Fiber Optical Probe Hydrophone, RP acoustic) was simultaneously conducted with the observation of the schlieren method.

Figure 6 shows a pressure profile obtained at a distance of $23 \mathrm{~mm}$ from the discharge point using the FOPH 2000. In the figure, the experimental data within $2.5 \mu$ s are affected by the flash noise of the electric discharge. We observed the $1 \mathrm{st} \mathrm{SW}$ of about $9.5 \mathrm{MPa}$ at $15.4 \mu$ s and the SHW of about $-7.4 \mathrm{MPa}$ at $24.4 \mu \mathrm{s}$. As indicated by the red arrows, there are some pressure fluctuations within the area between the 1st SW and SHW. According to the observation in Fig. 5 (3), it is thought that they show the pressure records of rebound shock waves generated by the collapses of air bubbles attaching to the silicone bag from the beginning when exposed to the 1st SW. The pressure variations after $30 \mu$ s are due to the motion of the cavitation bubbles. On the other hand, we should note the accuracy of the negative pressure of the SHW since the pressure data measured by the FOPH 2000 is estimated using the Tait equation. The density $\rho$ in the tensile region could be obtained to about $996.2 \mathrm{~kg} / \mathrm{m}^{3}$ when the atmosphere $P_{0}=1.01325 \times 10^{5} \mathrm{~Pa}$, the negative pressure, $P_{\mathrm{n}}=-7.4 \times 10^{6} \mathrm{~Pa}$, and the density at the atmosphere $\rho_{0}=999.7 \mathrm{~kg} / \mathrm{m}^{3}$. 


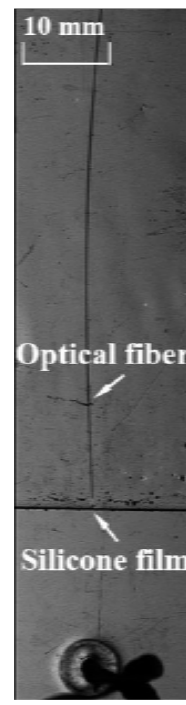

(1) $2.87 \mu \mathrm{s}$

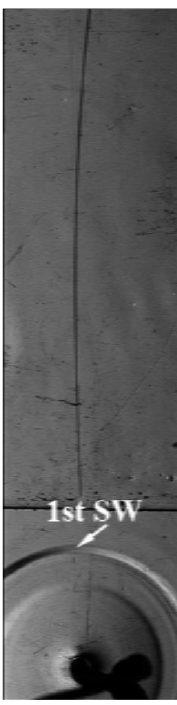

(2) $10.87 \mu \mathrm{s}$

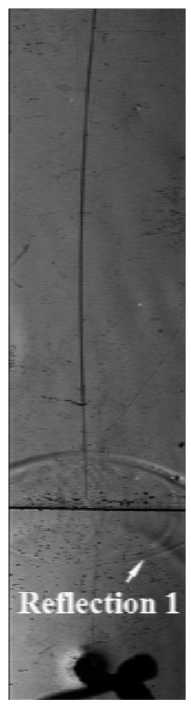

(3) $20.87 \mu \mathrm{s}$

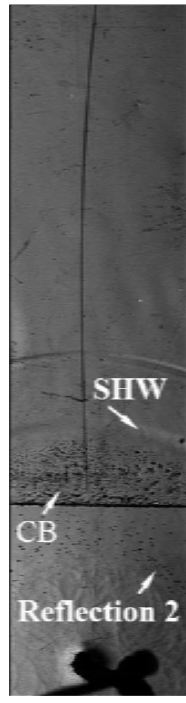

(4) $30.87 \mu \mathrm{s}$

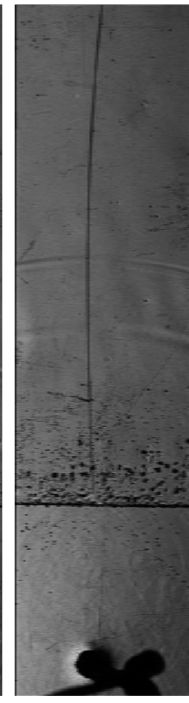

(5) $40.87 \mu \mathrm{s}$

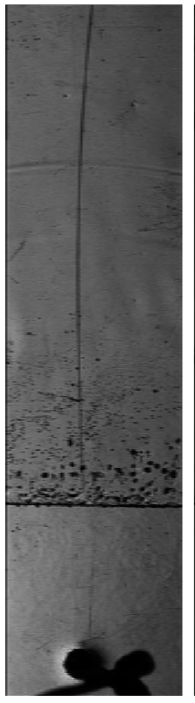

(6) $50.87 \mu \mathrm{s}$

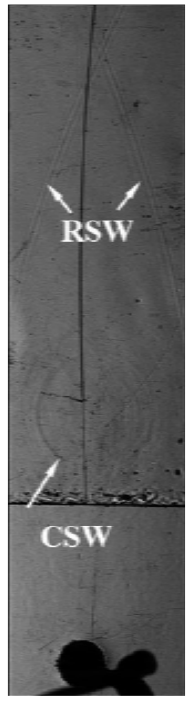

(7) $170.87 \mu \mathrm{s}$

Fig. 5 Observation of multiple waves generated by underwater electric discharge in narrow w ater chamber using schlieren method

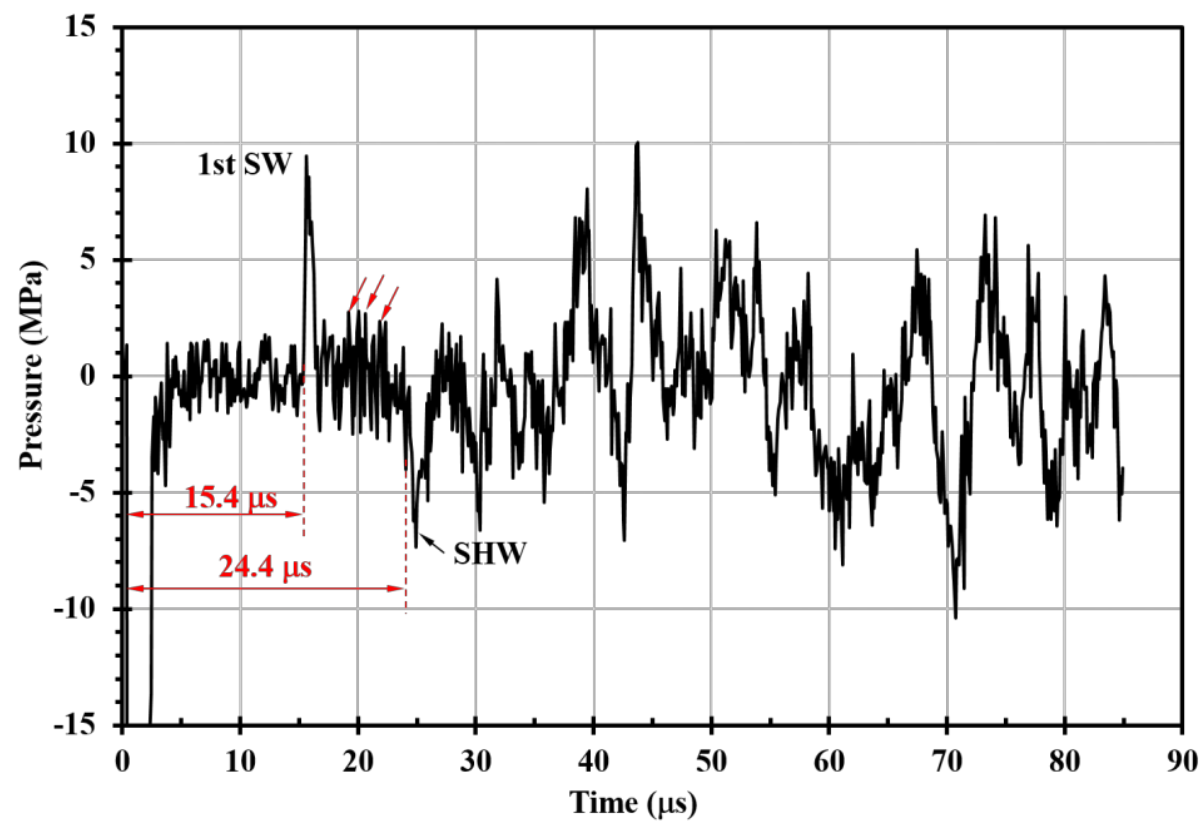

Fig. 6 Pressure profile obtained using FOPH 2000 at position of about $23 \mathrm{~mm}$ from discharge point

Figure 7 shows estimates of the number of viable cells for an electric discharge of $28.6 \mathrm{kV}$. The initial concentration of marine bacteria was about $5.37 \times 10^{4} \mathrm{cfu} / \mathrm{ml}$. The plots shown in this figure are of the averages for six sets of the bio-experimental data. Here, the error bars are not shown because the values of the standard deviation (STD) are too small to be clearly recognized in the exponential ordinate of the viability ratio. In the figures including the following bioexperimental results, the STD are less than 11 , even close to 0 except the point (STD $\approx 22$ ) at the beginning of the experiments. The solid squares are the reference data obtained from the cell suspension without the electric discharges. The number of viable cells hardly changes throughout the experiment. To attain the sterilization effect of only these cavitation bubbles, an air layer of 2 $\mathrm{mm}$ was set to prevent underwater shock wave entering the upper side of silicone film, as shown 
in the figure. The solid triangles and diamonds represent the results of the bio-experiments obtained with and without an air layer, respectively. The results show that after $4 \mathrm{~min}$, all of the marine bacteria are completely inactivated without air layer, while only one order of the number of marine bacteria are inactivated with a 2-mm air layer. It indicates the interaction of the cavitation bubble with the shock pressures enhance the sterilization effects. On the other hand, the solid triangle data indicate a tendency that the inactivation rate is slow but the number of viable cells is definitely reduced just by those oscillating bubbles. Their motions are also captured as shown in Fig. 8. The frame rate of the high-speed camera was $300 \mathrm{kfps}$. From Fig. 8, it is observed that the bubble motions are induced successively by interaction with the rebound shock waves of other bubbles. As a result, a shock pressure leading to the collapse of the bubble plays an important role in a high sterilization effect.

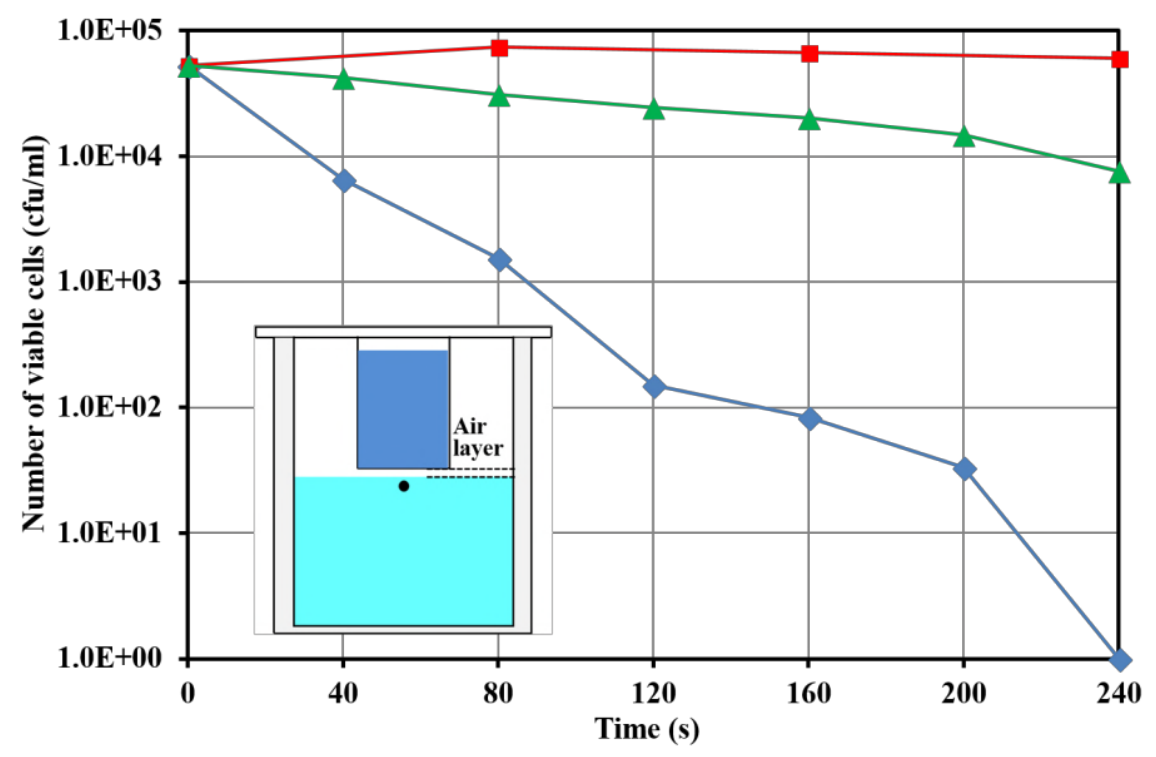

Fig. 7 Estimation of sterilization effect obtained with 28.6-kV electric discharges:

reference data, $\Delta$ with 2-mm air layer, and $>$ without air layer

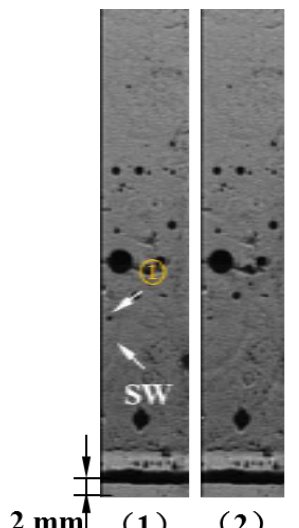

$2 \mathrm{~mm}$ (1)

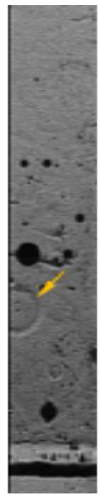

(3)

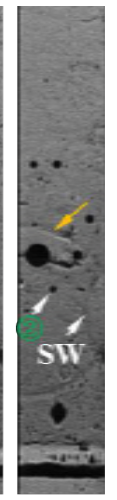

(4)

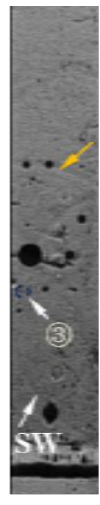

(5)

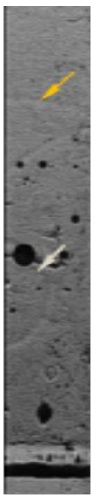

(6)

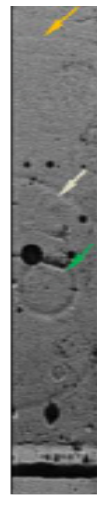

(7)

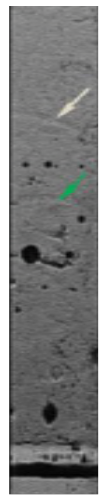

(8)

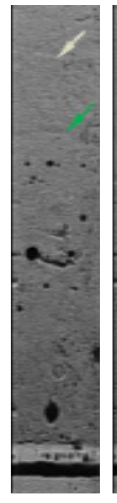

(9)

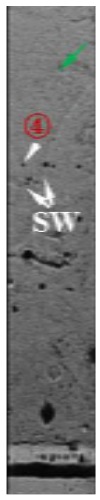

(10) (11)

Fig.8 Sequential images of collapses of cavitation bubbles with 2-mm air layer: the interval time is $3.33 \mu \mathrm{s}$

Figure 9 shows estimation on the number of viable cells under different output powers of the electric discharges. The solid triangles, diamonds, and circles represent the results obtained with an electric discharge of $31.6 \mathrm{kV}, 28.6 \mathrm{kV}$, and $18.8 \mathrm{kV}$, respectively. From Fig. 9, it can be seen that the times to obtain perfect inactivation are $160 \mathrm{~s}$ for $31.6 \mathrm{kV}$ and $240 \mathrm{~s}$ for $28.6 \mathrm{kV}$, while about two orders of the number of marine bacteria were inactivated after $240 \mathrm{~s}$ for $18.8 \mathrm{kV}$. The sterilization 
effect obviously increases with the output power of the electric discharge. It goes without saying that a larger output power of the electric discharge makes underwater shock waves stronger. In addition, the instant release of the discharge energy can deform the wall material and spread the width between the walls. Consequently, water in the narrow chamber is instantly stretched with the wall deformation, and a larger number of cavitation bubbles are also generated. On the other hand, as mentioned in Fig. 3, marine bacteria are inactivated by the mechanical action of rebound shock wave and the biochemical action of free radicals produced in the cavitation-shock interaction process. To investigate their respective sterilization effect, the sodium L-ascorbic was put into the cell suspension to remove the effect of free radicals. The results at $31.6-\mathrm{kV}$ electric discharges with the sodium L-ascorbic are presented by the solid squares. These data show that the number of marine bacteria barely changes during the experiment. It indicates that marine bacteria are not inactivated by the mechanical action of the bubble motion, and free radicals take mainly responsible for the inactivation in the present experimental setup. According to our previous study $(10,25)$, it has been found that the strong rebound shock waves are not generated in the present experimental chamber. In addition, considering the experimental results with an air layer of $2 \mathrm{~mm}$ in Fig. 7, all of the marine bacteria are also inactivated by the bio-chemical action of free radicals.

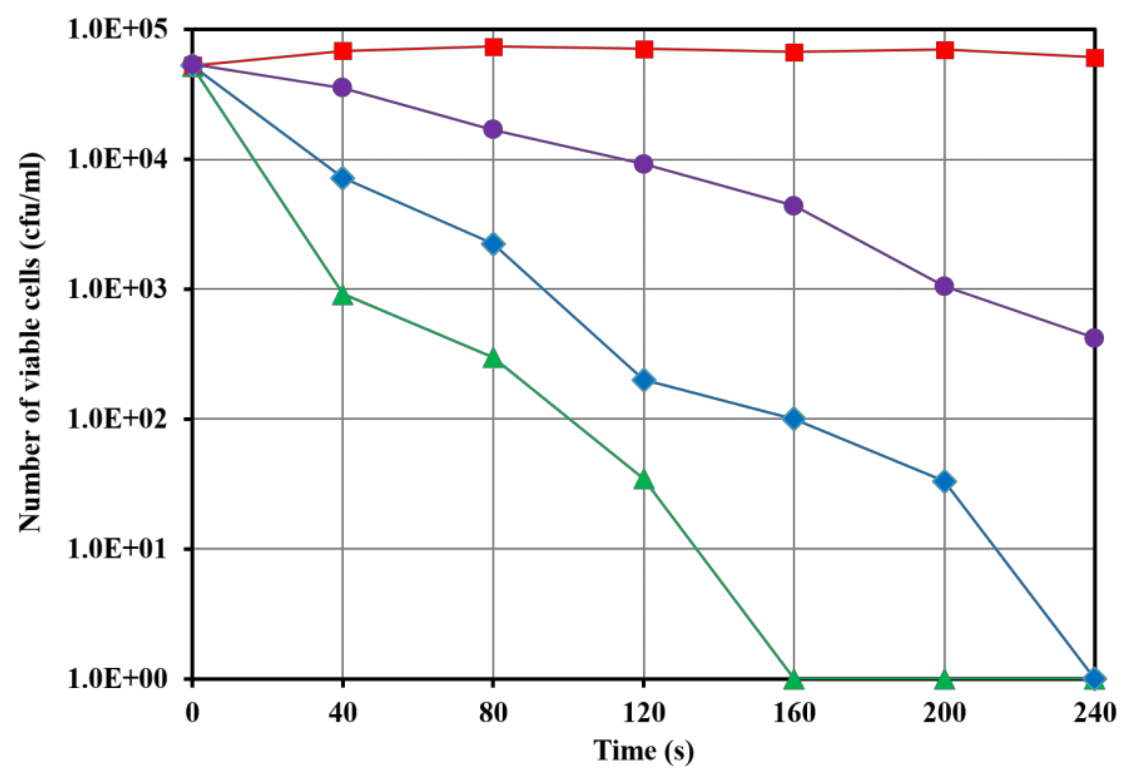

Fig. 9 Estimation of number of viable cells obtained with electric discharges of $31.6 \mathrm{kV}$ to 18.8 $\mathrm{kV}: \triangle 31.6 \mathrm{kV}$, and $\diamond 28.6 \mathrm{kV}, 18.8 \mathrm{kV}$, and $\square$ with sodium L-ascorbic

Free radicals inactivating marine bacteria are generated inside the bubble due to extremely high temperature and pressure, and then transfer to the outside through a boundary layer. Among the production of the chemical processes, hydroxyl $(\mathrm{OH})$ radicals are thought to be dominant specie due to their highly oxidative ability. However, it is difficult to directly measure or observe the $\mathrm{OH}$ radicals owing to their fast reaction in a dynamic stimulus (26). To examine the generation of the $\mathrm{OH}$ radicals in the present study, a digital pack tester $\left(\mathrm{DPM}-\mathrm{H}_{2} \mathrm{O}_{2}\right.$, Kyoritsu Chemical-Check Lab., Corp.) were used to measure the concentration of the $\mathrm{H}_{2} \mathrm{O}_{2}$, one of the productions of their chemical reactions. Its measuring range is from 0.10 to $2.0 \mathrm{mg} / \mathrm{L}$ and the resolution is $0.05 \mathrm{mg} / \mathrm{L}$. Figure 10 shows the concentration of the $\mathrm{H}_{2} \mathrm{O}_{2}$ with an electric discharge of $31.6 \mathrm{kV}$ to $18.8 \mathrm{kV}$. The measurements were conducted after the electric discharges of 5000, 8400, and 10200 shots. The symbols in the figure indicate of the averages for 5-set measurements that are all the same owing to the respectively small resolution of the digital pack tester. The results show that the concentration of $\mathrm{H}_{2} \mathrm{O}_{2}$ increases with the output power of the electric discharge. It indicates an increase in the strength of the cavitation-shock interaction. In the case of a 2-mm air layer, the concentration of the 
$\mathrm{H}_{2} \mathrm{O}_{2}$ is detected to about $0.5 \mathrm{mg} / \mathrm{L}$ after 10020-shot electric discharges, so that marine bacteria are inactivated only by those oscillating bubbles, but a high sterilization effect is not obtained. The results of the measurements show good agreements with the bio-experimental results.

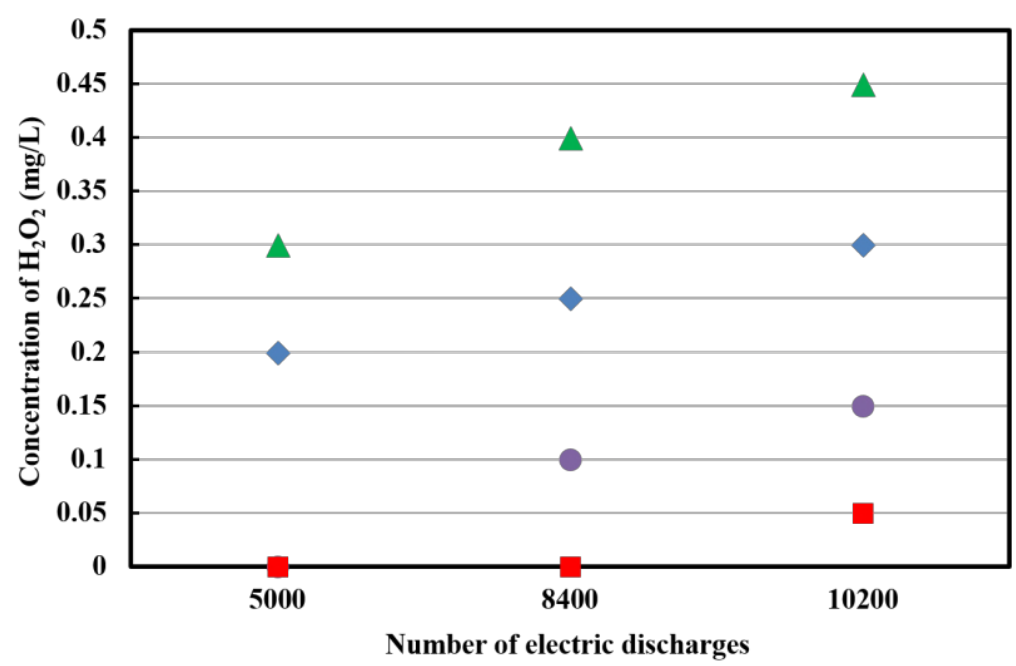

Fig. 10 Estimation of concentration of $\mathrm{H}_{2} \mathrm{O}_{2}$ using digital pack tester with electric discharge of $31.6 \mathrm{kV}$ to $18.8 \mathrm{kV}$ :

$\triangle 31.6 \mathrm{kV}$, and $\diamond 28.6 \mathrm{kV}, 18.8 \mathrm{kV}$, and $\square$ with 2-mm air layer

Based on the pressure measurements, the pressure of reflected shock waves are only dozens of atmospheres, even a few atmospheres in the case of the air layer in the narrow water chamber. However, we obtain a high sterilization effect using the chemical action of the $\mathrm{OH}$ radicals in the cavitation-shock interaction, as shown in Fig. 9. To estimate the condition for generation of the $\mathrm{OH}$ radicals, a theoretical analysis was carries out by solving the bubble dynamic model. First of all, the theoretical solution of the Rayleigh-Plesset equation for the growth of a 5- $\mu$ m radius bubble nucleus is shown in Fig. 11. In this analysis, the density of water $\rho_{\infty}=999.7 \mathrm{~kg} / \mathrm{m}^{3}$, the viscosity coefficient $\mu=1.307 \times 10^{-3} \mathrm{~Pa} \cdot \mathrm{s}$, the surface tension $\sigma=74 \times 10^{-3} \mathrm{~N} / \mathrm{m}$, the density of gas $\rho_{\mathrm{g}}=1.20 \mathrm{~kg} / \mathrm{m}^{3}$ at $T_{0}=293.15 \mathrm{~K}$ when $R=R_{0}$, the thermal conductivity in the gas $\lambda_{g}=0.599 \mathrm{~W} /(\mathrm{m} \cdot \mathrm{K})$, the heat capacity of gas $C_{\mathrm{p}}=4186 \mathrm{~J} /(\mathrm{kg} \cdot \mathrm{K})$, the atmospheric pressure $P_{0}=1.01325 \times 10^{5} \mathrm{~Pa}$ were used. The initial total number of the molecules inside the bubble was determined by Eqs. (11) and (12) when $P_{\text {in }}=1.03 \times 10^{5} \mathrm{~Pa}, T_{\text {in }}=293.15 \mathrm{~K}, R=5 \mu \mathrm{m}$. The initial number of water vapor $N_{\text {water }}$ corresponded to the equilibrium density at the wall, $C_{\mathrm{r}}$. The difference between the number of total molecules and water vapor was defined as the number of other molecules, $N_{\text {others. }}$ In this figure, the blue lines and orange lines indicate the results obtained with and without the mass transportation, respectively. For the variation of the bubble radius indicated by the solid lines, the bubble nucleus grows to a larger size, about $13 \mu \mathrm{m}$ in radius in the case of the transportation of water vapor at the interface. Correspondingly, the minimum internal pressure is $0.077 \times 10^{5} \mathrm{~Pa}$, slightly higher than $0.069 \times 10^{5}$ $\mathrm{Pa}$ when not considering the mass transportation due to the energy loss of transferring water vapor, as shown by the dashed lines. Furthermore, the minimum internal pressure slightly increases at the second expansion. Hence, the pressure difference between inside and outside of the bubble shows the largest at the first expansion, so that maximum values of temperature and pressure in the bubble are obtained when the oscillating bubble is exposed to a shock pressure. As a result, the motion phases of the bubble during the first expansion in Fig. 11 were substituted into the impact model in order to analyze the interaction of a bubble with a shock pressure. 


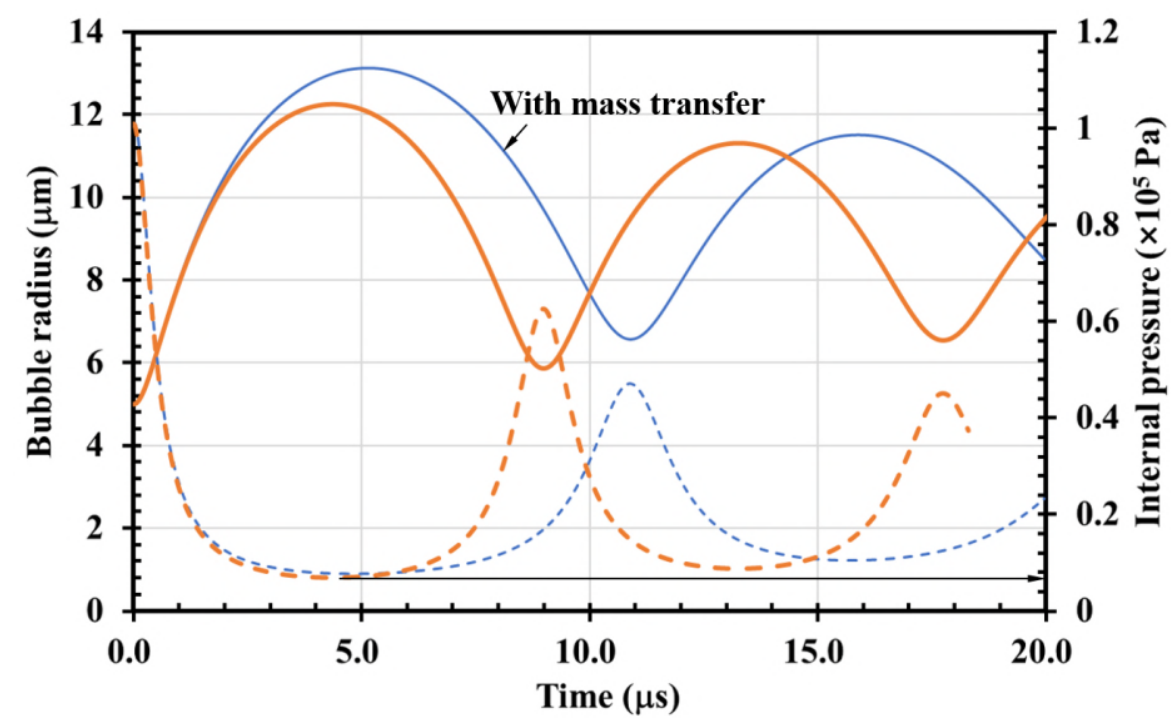

Fig. 11 Oscillation of 5- $\mu \mathrm{m}$ radius bubble nucleus solved by Rayleigh-Plesset equation with and without mass transportation

In the present water chamber, there are possibilities that shock pressures interact on oscillating bubbles in arbitrary phases as to radius, surface velocity, and internal pressure. To obtain the collapsing motions of these bubbles, the Herring equation was solved in consideration of heat conductivity and mass transfer in the impact model. The theoretical solution is shown in Fig. 12. The abscissa indicates the time corresponding to the initial bubble motion phases in Fig. 11 , and the ordinate is the peak internal temperature of the bubble obtained when the oscillating bubble in this motion phase is exposed to a shock wave of $P_{\text {shock }}=2 \mathrm{MPa}$. The pressure of 2-MPa is the average of reflected shock wave at $28.6-\mathrm{kV}$ electric discharge. In the figure, it can be seen that the peak internal temperature is the smallest value of about $1400 \mathrm{~K}$ at the starting point; $R_{0}=$ $5 \mu \mathrm{m}, u=0 \mathrm{~m} / \mathrm{s}, T_{\mathrm{in}}=293.15 \mathrm{~K}$, and $P_{\mathrm{in}}=1.01 \times 10^{5} \mathrm{~Pa}$. During the expanding phase of the bubble, the peak internal temperature increases due to violent bubble motion induced by a large pressure difference between inside and outside of the bubble, $\Delta P_{s-i}=P_{\text {shock }}-P_{\text {in, }}$ and reaches a maximum value of about $7000 \mathrm{~K}$ at $R_{0}=13 \mu \mathrm{m}, u \approx 0 \mathrm{~m} / \mathrm{s}, T_{\text {in }}=293.15 \mathrm{~K}$, and $P_{\text {in }}=0.077 \times 10^{5} \mathrm{~Pa}$. When the shock pressure interacts with a contracting bubble, it is found that the internal peak pressure decreases to about $2400 \mathrm{~K}$ at $R_{0}=6.59 \mu \mathrm{m}, u \approx 0 \mathrm{~m} / \mathrm{s}, T_{\text {in }}=293.15 \mathrm{~K}$, and $P_{\text {in }}=0.48 \times 10^{5} \mathrm{~Pa}$. On the other hand, we should note the conditions of the bubble just when exposed to the shock wave. The radius, surface velocity, and internal pressure of bubble mainly influence the surface tension, $2 \sigma / R_{0}$, the kinetic energy, $\rho 1 u^{2}$, and the pressure difference between outside and inside of the bubble, $\Delta P_{s-i}$. Here, the $\rho 1 u^{2}$ and $2 \sigma / R_{0}$ range within $0-0.12 \times 10^{5} \mathrm{~Pa}$ and $0.11 \times 10^{5} \mathrm{~Pa}-$ $0.29 \times 10^{5} \mathrm{~Pa}$ during the first expansion of the bubble, as shown in Fig. 11. Compared with the value of the pressure difference, the surface tension and kinetic energy value slightly works on the calculation of the peak internal pressure. As a result, it is obviously concluded that the pressure difference, $\Delta P_{s-i}$, is a main factor on the collapse of the bubble when exposed to a shock pressure. 


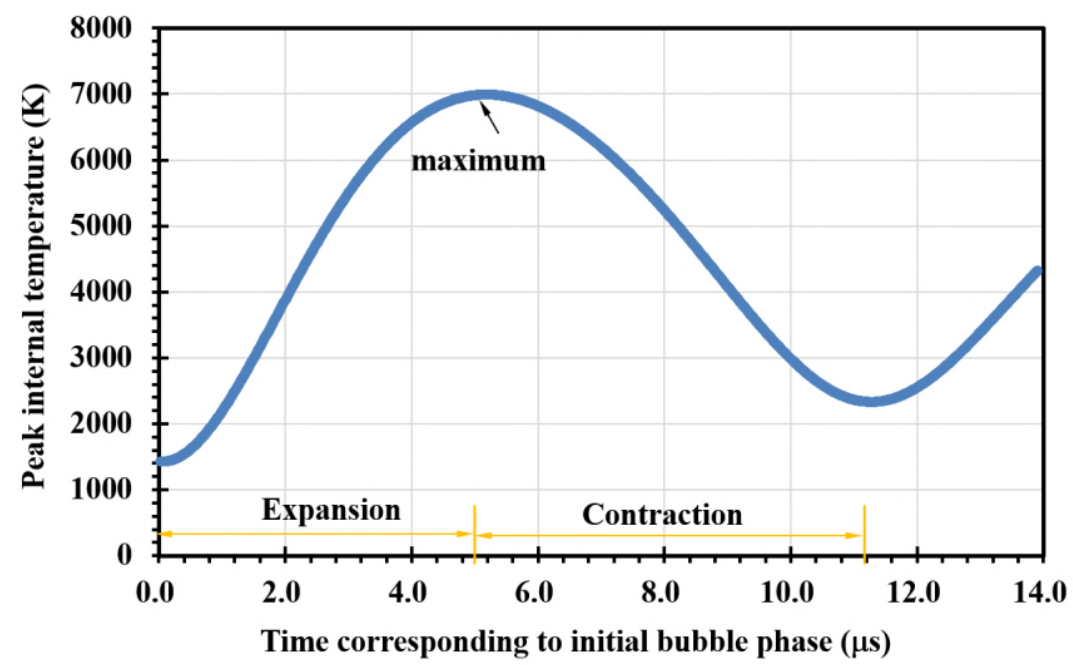

Fig. 12 Peak temperatures inside bubbles corresponding to initial states interacting shock pressure of $2 \mathrm{MPa}$

From the given experimental observation, the pressures leading to the collapses of oscillating bubbles are mainly from the reflected shock waves on the chamber wall or the transmission of the elastic wave travelling in the wall material. The experimental pressure measurements indicated that the pressure ranges of shock waves interacting with oscillating bubbles probably reach about dozens of atmospheres. Hence, we assumed that $P_{\text {shock }}$ ranges from $0.1 \mathrm{MPa}$ to $5.0 \mathrm{MPa}$ and analyzed theoretically the maximum internal temperatures and pressures generated by the collapse of bubbles exposed to the shock pressures. The results are shown in Fig. 13. The maximum of the peak internal temperatures as indicated by an arrow in Fig. 12 are represented as a maximum internal temperature at $P_{\text {shock }}=2 \mathrm{MPa}$ in Fig. 13. Corresponding maximum internal pressures are also solved by the bubble dynamic model. From Fig. 13, we can see that the maximum internal temperatures and pressures produced by the cavitation-shock interactions are estimated to be over $1200 \mathrm{~K}$ and $50 \mathrm{MPa}$ in the range of $P_{\text {shock }}=0.1$ to $5.0 \mathrm{MPa}$, respectively. As described in the study of Wang et al. (17), the $\mathrm{OH}$ radicals could be generated at the internal temperature of bubble, above $1000 \mathrm{~K}$. Incidentally, the condition of $0.1-\mathrm{MPa}$ is equivalent to the experimental condition of a 2-mm air layer. In this case, underwater shock waves are prevented to entering the upper side over a silicone film. However, cavitation bubbles are still produced due to the shear waves propagating in the wall material. During the growth of the bubble nuclei, it is thought that the ambient pressure around them could return rapidly to atmosphere. Hence, we assumed that the oscillating bubbles are suddenly exposed to a $0.1-\mathrm{MPa}$ ambient pressure. Consequently, as shown in Fig. 10, we obtained the generation of the $\mathrm{OH}$ radicals in evidence, but it is difficult to say that we could indicate significant results in the experiment under the condition of an air layer. On the other hand, from Fig.13, the maximum temperature and pressure in a bubble increase with the increase of the shock pressure and reach about $10800 \mathrm{~K}$ and $15600 \mathrm{MPa}$ at $P_{\text {shock }}=5 \mathrm{MPa}$, respectively, so that the concentration of the $\mathrm{H}_{2} \mathrm{O}_{2}$ is probably increased with increase of the inducing shock pressures and leads to a higher sterilization. Those results are supported by the experimental results indicated in Figs. 9 and 10. Hence, the generation of the $\mathrm{OH}$ radicals can be obtained at $P_{\text {shock }}=0.1 \mathrm{MPa}$, and their concentration increases with increase of the shock pressure in the cavitation-shock interaction. The theoretical solutions obtained with the bubble dynamic model are consistent with the bio-experimental results and concentration measurements. 


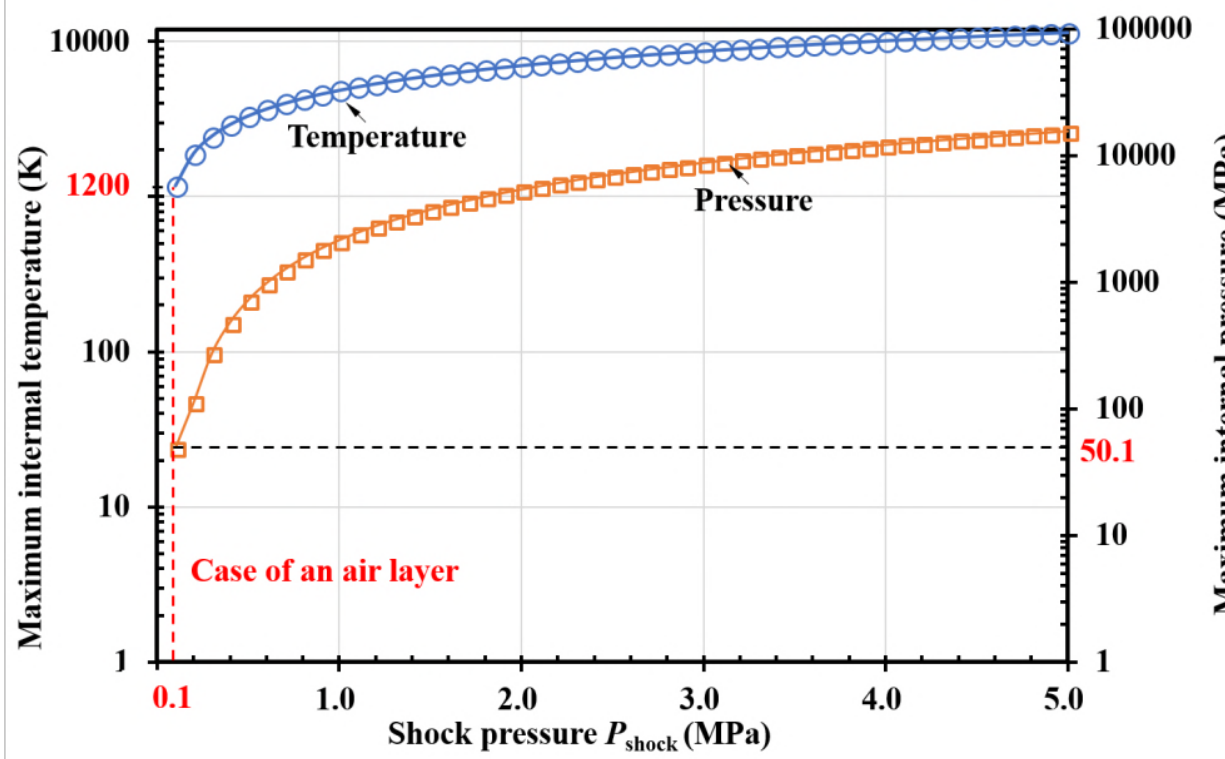

Fig. 13 Theoretical solution for interaction of oscillating bubble with shock pressure of 0.1 $\mathrm{MPa}$ to $5 \mathrm{MPa}$

\section{Summary}

Sterilization effects of the cavitation-shock interaction were investigated by a bio-experiment of marine Vibrio sp. in the narrow water chamber. Cavitation bubbles were generated by a tensile force acting on water due to the shear waves travelling in the window material as a result of instant release of electric discharge energy. The observation of the schlieren method showed that the collapses of those cavitation bubbles were induced by the underwater shock wave, and their interaction has a potential to inactivate marine bacteria. In the bio-experiment, an air layer was set to prevent the underwater shock waves entering the cell suspension, so that the sterilization effects of only cavitation bubble were obtained. It was found that all of marine bacteria were perfectly inactivated in several minutes in the case of the cavitation-shock interaction while only one order of bacteria was inactivated with an air layer. It indicated that a high sterilization requires a strong shock pressure leading to violent collapses of cavitation bubbles. Furthermore, the sterilization effects increase with the output power of the electric discharge since both of the shock pressures and the number density of cavitation bubbles increase.

On the other hand, the inactivation of marine bacteria was proven to mainly depend on the biochemical action of free radicals, by adding sodium L-ascorbic to the cell suspension. To clarify the generation of the $\mathrm{OH}$ radicals, we measured the concentration of the $\mathrm{H}_{2} \mathrm{O}_{2}$ under different conditions of the electric discharges. The concentration of the $\mathrm{H}_{2} \mathrm{O}_{2}$ increased with the output powers. It suggested that a strong strength of the cavitation-shock interaction produces a great number of oxidative radicals, and thus leads to a high inactivation of marine bacteria. The measurements showed good agreements with the bio-experiments.

In order to estimate the condition for generating the $\mathrm{OH}$ radicals, a model of the bubble dynamics was developed consisting of an oscillation model for the growth of bubble nuclei and an impact model to describe the interaction of a cavitation bubble with a shock pressure. The transfer of heat and water vapor were also considered in the model. The theoretical analysis was carried out under the conditions of the present experiments. The theoretical solutions showed that the pressure difference between inside and outside of a bubble is a main factor in affecting its collapsing motion. It was also found that the internal temperature and pressure could achieve the 
condition for the generation of the $\mathrm{OH}$ radicals in the case of using an air layer and increase with the inducing shock pressure, so that a higher sterilization effect was obtained, identically as described in the bio-experiments and the concentration measurements.

\section{Author Contributions}

J.W. wrote the main text, and made the bio-experiments and calculation of the bubble dynamic model with the help of T.K. and M.S.. A.A. and Y.W. checked and corrected the work. Furthermore, Yiwei Wang has the idea of this work. C.H. helped in the proper organization of the calculations.

\section{Acknowledgements}

This work was supported by the National Natural Science Foundation of China, grant numbers 11772340 and 11332011, and the JSPS KAKENHI, grant Numbers 16H04600 and 16K14512. In addition, we would like to sincerely thank Nac Image Technology Inc. for the supply of optical experimental equipment.

\section{Reference}

1. Escaler, X., E. Egusquiza, M. Farhat, F. Avellan, M. Coussirat, 2006. Detection of cavitation in hydraulic turbines. Mechanical Systems and Signal Processing. 20: 983-1007.

2. Arndt, R. E., 1981. Cavitation in fluid machinery and hydraulic structures. Annual Review of Fluid Mechanics. 13: 273-326.

3. Thapa, B. S., B. Thapa, O. G. Dahlhaug, 2012. Empirical modelling of sediment erosion in Francis turbines. Energy. 41: 386-391.

4. Wu, S., Z. Zuo, H. A. Stone, S. Liu, 2017. Motion of a Free-Settling Spherical Particle Driven by a Laser-induced Bubble. Physical Review Letters 119: 084501

5. Takayama, K., 1993. Application of Underwater Shock Wave Focusing to the Development of Extracorporeal Shock Wave Lithotripsy. Japanese Journal of Applied Physics. 32: 2192-2198.

6. Coussios, C. C., R. A. Roy, 2008. Applications of acoustics and cavitation to noninvasive therapy and drug delivery. Annual Review of Fluid Mechanics. 40: 395-420.

7. Song, W. D., M. H. Hong, B. Lukyanchuk, C. T. Chong, 2004. Laser-induced cavitation bubbles for cleaning of solid surfaces. Journal of Applied Physics. 95: 2925-2956.

8. Loske, A. M., U. M. Alvarez, C. Hernández-Galicia, E. Castaño-Tostado, F. E. Prieto, 2002. Bactericidal effect of underwater shock waves on Escherichia coli ATCC 10536 suspensions. Innovative Food Science \& Emerging Technologies. 3: 321-327.

9. Abe, A., H. Mimura, 2013. Sterilization of Ships' Ballast Water. In Bubble Dynamics and Shock Waves, SHOCK WAVES 8. Springer Berlin Heidelberg, pp. 339-362.

10. Wang, J., A. Abe, 2016. Experimental Verification of Shock Sterilization for Marine Vibrio sp. using Microbubbles Interacting with Underwater Shock Waves. Journal of Marine Science and Technology. 21: 679-688.

11. Koita, T., K. Hayashi, M. Sun, 2015. Experimental Study of Underwater Shock Wave and Cavitation Generated by Underwater Electric Discharge in a Narrow Container. Proceedings of the 29th International Symposium on Shock Waves 2, pp.1505-1510.

12. Wang, J., A. Abe, T. Koita, M. Sun, 2017, Contribution of cavitation generation to shock wave sterilization effects in a narrow water chamber, Proceedings of 31th International Symposium on Shock Waves, No. SBM000165, 2017, 1-6, in a USB memory.

13. Sankin, G. N., W. N. Simmons, S. L. Zhu, P, Zhong, 2005. Shock Wave Interaction with Laser-Generated Single Bubbles. Physical Review Letters. 95: 034501.

14. Sankin, G. N., P. Zhong, 2006. Interaction between shock wave and single inertial bubbles near an elastic boundary. Physical Review E. 74: 046304. 
15. Iloreta, J. I., N. M. Fung, A. J. Szeri, 2008. Dynamic of bubble near a rigid surface subjected to lithotripter shock wave: Part 1. Consequences of interference between incident and reflected waves. Journal of Fluid Mechanics. 616: 43-61

16. Klaseboer, E., S. W. Fong, C. K. Turangan, B. C. Khoo, A. J. Szeri, M. Calvisi, G. N. Sankin, P. Zhong, 2007. Interaction of lithotripter shockwaves with single inertial cavitation bubbles. Journal of Fluid Mechanics. 593: 33-56.

17. Wang, J., A. Abe, Y. Wang, C. Huang, 2018. Fundamental study of sterilization effects on marine Vibrio sp. in a cylindrical water chamber with supply of only underwater shock waves. Ultrasonics Sonochemisty. 42: 541-550.

18. Brenner, M. P., S. Hilgenfeldt, D. Lohse, 2002. Single-bubble sonoluminescence. Reviews of Modern Physics. 74: 425-483.

19. Toegel, R., B. Gompf, R. Pecha, D. Lohse, 2000. Does water vapor prevent upscaling sonoluminescence. Physical Review Letters. 85: 3165-3168.

20. Toegel, R., S. Hilgenfeldt, D. Lohse, 2002. Suppressing dissociation in sonoluminescing bubbles: the effect of excluded volume. Physical Review Letter. 88: 034301.

21. Yasui, K., K. Kato, 2012. Bubble dynamics and sonoluminescence from helium and xenon in mercury and water. Physical Review E. 86: 036320.

22. Toegel, R., D. Lohse, 2002. Phase diagrams for sonoluminescing bubbles: a comparison between experiment and theory. Journal of Chemistry Physics. 118: 1863-1875.

23. Dular, M., O. Coutierdelgosha, 2013. Thermodynamic effects during growth and collapse of a single cavitation bubble. Journal of Fluid Mechanics. 736: 44-66.

24. Herring, C., 1941. Theory of the pulsations of the gas bubble produced by an underwater explosion. Columbia Univ., Division of National Defense Research.

25. Wang, J., A. Abe, 2016. A hybrid analytical model of sterilization effect on marine bacteria using microbubbles interacting with shock wave. Journal of Marine Science and Technology. 21: 385-395.

26. Takahashi, M., K. Chiba, P. Li, 2007. Free-radical generation from collapsing microbubbles in the absence of a dynamic stimulus. Journal of Physical Chemistry B. 111: 1343-1347.

Fig. 1 Observation of multiple waves generated by underwater electric discharge with 2-mm air layer in Ref. (12). ELW: Elastic wave, SW: Shock wave, RSW1: Reflection of shock wave, RSW: Reflection of 2nd wave, TSW: Transition of shock wave through air layer, and CB: Cavitation bubbles.

Fig. 2 Schematic of bio-experimental setup for estimating sterilization effects

Fig. 3 Schematic of inactivation mechanism of cavitation-shock interaction generated in narrow water chamber: (a) Schematic of narrow water chamber, (b) Collapses of cavitation bubbles, and (c) Photo of marine Vibrio sp.

Fig. 4 Schematic of bubble dynamic model for describing growth of bubble nuclei and cavitationshock interaction

Fig. 5 Observation of multiple waves generated by underwater electric discharge in narrow water chamber using schlieren method

Fig. 6 Pressure profile obtained using FOPH 2000 at position of about $23 \mathrm{~mm}$ from discharge point Fig. 7 Estimation of sterilization effect obtained with 28.6-kV electric discharges: $\square$ reference data,

$\Delta$ with 2-mm air layer, and $\diamond$ without air layer

Fig.8 Sequential images of collapses of cavitation bubbles with 2-mm air layer: the interval time is $3.33 \mu \mathrm{s}$

Fig. 9 Estimation of number of viable cells obtained with electric discharges of $31.6 \mathrm{kV}$ to $18.8 \mathrm{kV}$ : $\triangle 31.6 \mathrm{kV}$, and $\diamond 28.6 \mathrm{kV}, 18.8 \mathrm{kV}$, and $\square$ with sodium L-ascorbic

Fig. 10 Estimation of concentration of $\mathrm{H}_{2} \mathrm{O}_{2}$ using digital pack tester with electric discharge of 31.6 
$\mathrm{kV}$ to $18.8 \mathrm{kV}: \triangle 31.6 \mathrm{kV}$, and $28.6 \mathrm{kV}, 18.8 \mathrm{kV}$, and $\square$ with $2-\mathrm{mm}$ air layer

Fig. 11 Oscillation of 5- $\mu \mathrm{m}$ radius bubble nucleus solved by Rayleigh-Plesset equation with and without mass transportation

Fig. 12 Peak temperatures inside bubbles corresponding to initial states interacting shock pressure of $2 \mathrm{MPa}$

Fig. 13 Theoretical solution for interaction of oscillating bubble with shock pressure of $0.1 \mathrm{MPa}$ to $5 \mathrm{MPa}$ 